\title{
LA CARTE GEOGRAPHIQUE ET LES ELECTIONS
}

\author{
Par \\ Matezo Bakunda \\ Professeur Matezo Bakunda est Directeur Général Adjoint \\ de l'Institut Géographique du Congo
}

\section{INTRODUCTION}

Le terme election vient du latin electio du verbe eligere: choisir, élire. Election signifie donc essentiellement un choix, une désignation d'une ou de plusieurs personnes par voie de suffrages. Les élections peuvent être libres ou contraignantes, administratives, législatives ou présidentielles. Les élections se présentent sous plusieurs formes; majoritaires, représentations proportionnelles, systèmes mixtes, etc....

Une opération électorale sans référence aux principes élémentaires de la comptabilité des habitants peut engendrer des dysfonctions au sein de l'appareil politico-administratif de l'Etat. C'est ainsi que généralement des données du recensement avec un support cartographie.

Les élections sont des opérations de grande importance pour chaque pays. Elles constituent la forme la plus démocratique pour exprimer et accéder au pouvoir. Elles sont la source d'une série de données de base pour l'administration ainsi que pour l'orientation de la politique économique et sociale d'un pays et fournissent un point de référence aux politiciens, un cadre de décision si elles s'opèrent normalement et une source de paix, de bonheur pour le pays concerné.

L'importance des élections pour le choix des dirigeants a été reconnue depuis longtemps. Dans de nombreux pays, l'organisation des élections est une tradition vieille de plusieurs années. Aucun pays ne nie l'intérêt qu'il y a à choisir ses dirigeants par cette voie. De nombreuses recommandations internationales soulignent jusqu'à ce jour le rôle considérable des élections et proposent des méthodes susceptibles d'étendre leur portée, d'accroître leur validité et d'améliorer en même temps que leur valeur propre, la comparabilité internationale dans ce domaine.

La plupart des pays du monde sont convaincus et pensent que les élections constituent la voie indiquée pour le choix des dirigeants. Si les opérations se déroulent sans beaucoup de heurts dans le pays développés, dans les pays du tiers monde par contre, et particulièrement ceux d'Afrique, beaucoup d'efforts restent à faire. On a toujours dénoncé le truquage, la fraude, la non transparence dans l'organisation de leurs élections. Et pourtant, l'expérience de bonnes élections a montré que c'est là la voie absolument indispensable à la Bonne Gouvernance d'un pays. 


\section{De L'organisation Des Elections}

L'organisation des élections requiert le dénombrement au préalable de toute la population, ressortissant du pays dans lequel l'opération a lieu et répartit par entité administrative. Cette connaissance de la population permet de déterminer le corps électoral pour l'ensemble du pays par entité administrative et le nombre des sièges à pourvoir par circonscription électorale. En plus, la réalisation des élections exige au préalable l'enrôlement de la population électorale dont l'âge devra être défini.

En mettant au point l'organisation et l'administration des élections, il importe de bien définir les attributions et les rapports de services chargés de leur tenue et les seservices qui ser ont consultés.

L'institution d'un bureau ou d'une Commission Nationale Electorale Indépendante présente des avantages considérables et constitue le cadre à partir duquel seront développés le programme et les travaux préliminaires des futures élections. Il faut donc au départ cette Commission Electorale.

Sa permanence rend possible la formation d'un personnel technique éprouvé, la constitution et la mise à jour des fiches contenant des renseignements statistiques et géographiques essentiels. On peut adjoindre à cette Commission, un bureau ou un Comité National des opérations préélectorales et électorales, et un secrétariat exécutif. Ce sont là deux structures d'aide et de soutien à la Commission Nationale Electorale.

\section{Les Travaux Preparatoires aux Elections}

Toute opération électorale quelle que soit le pays dans lequel elle a lieu comporte une série d'opérations préparatoires dont le nombre, l'ampleur, et la complexité varient d'un pays à l'autre en fonction des facteurs tels que:

- la nature du terrain (accidenté ou plate forme);

- le mode de répartition de la population (dispersée ou concentrée);

- sa composition sociale (un ou plusieurs groupes ethniques);

- son degré d'instruction;

- l'unité linguistique;

- l'importance des moyens de communication et de transports disponibles;

- l'exigence des renseignements récents et détaillés d'ordre statistiques, géographiques et cartographiques);

- l'efficacité de divers organes auxquels on pourrait faire appel pour la transparence et une réussite totale des opérations.

Les travaux préparatoires aux élections sont notamment, l'identification, le recensement et l'enrôlement. 


\section{L'IDENTIFICATION}

Cette opération consiste à trier la population d'une nationalité donnée en âge de voter d'écarter les opérations électorales pour les personnes plus jeunes et celles n'appartenant pas à la nationalité du pays.

Considérée comme telle, l'identification est une opération couplée avec l'enrôlement et les stratégies à mettre en œuvre seront différentes d'une région à l'autre compte tenu des spécificités de chacune et des contraintes de temps et des moyens dont on dispose.

A l'identification les variables à saisir sont notamment:

- le nom, le post-nom, le prénom;

- le sexe;

- le lieu de naissance;

- la date de naissance;

- l'adresse de la résidence actuelle (d'enrôlement);

- le $\mathrm{N}^{\circ}$ de bureau de recensement.

\section{Le RECENSEMENT}

Un recensement de population peut être défini comme l'ensemble des opérations qui consistent à recueillir, à grouper, et à publier des données démographiques, économiques et sociales se rapportant à un moment déterminé ou à certaines périodes données, à tous les habitants d'un pays ou territoire indiqué.

Le but visé par cette opération est de mettre à la disposition du pays des chiffres de population fiables et à jour en vue de déterminer:

- La population totale de droit c.à.d. celle qui réside habituellement au lieu du recensement permettant de calculer le nombre de sièges à pouvoir par circonscription électorale.

- La population électrice de fait c.à.d. celle qui est présente au lieu de recensement à la date de cette opération. Cette population sera enrôlée et répartie par entité administrative.

\section{L'ENROLEMENT}

C'est la dernière des opérations préélectorales. Elle consiste en l'inscription au rôle électoral de la population en âge de voter et qui aura été préalablement identifiée. L'enrôlement vise l'établissement des listes électorales où seront enregistrées les personnes appelées à voter.

Les informations à saisir à l'enrôlement sont mêmes que celles concernées par l'identification. Les renseignements à collecter seront consignés dans un registre 
où des cases sont prévues pour recevoir une empreinte digitale (à exploiter en cas de doute) et une photo passeport de l'enrôlement.

Toutes les trois opérations décrites ci-dessus sont fondamentales. Outre ces tâches et la préparation du texte sur la loi électorale, les opérations suivantes sont nécessaires et doivent être effectuées concomitamment avec les précédentes.

Il s'agit:

- des travaux géographiques et cartologiques;

- de la détermination des circonscriptions électorales;

- de l'établissement et de plébiscité en faveur des élections

\section{La Carte GeographiQue et L'organisation des Elections}

\section{Definition de la carte geographique}

Une carte géographique est la représentation plane conventionnelle schématisée de tout ou une partie de la surface terrestre suivant des rapports de similitude qu'on appelle échelle. Elle est un des meilleurs moyens de localisation de phénomènes terrestres, une forme d'expressions dont on sert habituellement dans plusieurs domaines de la vie nationale: plan, santé, éducation, économie, travaux publics, défense nationale, environnement et tourisme, urbanisme, agriculture, énergie et transport, etc.

La carte géographique est un support incontournable dans le programme de développement et de reconstruction d'un pays. Elle peut représenter:

- Des éléments physiques: les montagnes, les plaines, les végétations, le sol, les cours d'eau, les lacs, les marécages, etc.

- Des éléments humains: la densité de la population, les centres urbains, les voies de communication, les circonscriptions administratives; chefferies, secteurs, etc.

- Des éléments économiques: les ressources agricoles, minières et énergétiques, la pêche, les forêts, etc.

La carte géographique est aussi dans l'organisation des éléments dans l'organisation des élections un support indispensable auquel on devra obligatoirement recourir. Elle est dans le domaine électoral un des aspects les plus importants dans la gamme des possibilités de ses applications.

\section{La Carte Geographique Electorale}

Elle est un support par excellence des opérations électorales et publiées après promulgation de la loi électorale. Ce document permet de visualiser la situation 
générale sue le terrain, l'organisation générale, ce qui permet de corriger certains détails, de suivie des opérations dans tout leur déroulement.

La carte géographique électorale présente aux yeux des dirigeants en particulier et de la population en général de nombreux avantages:

Elle représente tout le territoire national soumis aux électeurs ainsi que son découpage en aires ou en districts électoraux. Il s'agit des espaces géographiques délimités dans lesquels vont se dérouler les opérations décrites ci-haut en tenant compte des contraintes comme le temps de collecte, la superficie, et la taille de la population. Chaque circonscription électorale pourra disposer à la rigueur de la carte de son territoire qui indiquerait les limites, les routes et autres moyens de communication, les villages et centres importants se trouvant en son sein. L'opération paraît certes ambitieuse et coûteuse, mais les avantages que l'on en tire valent l'effort et l'argent investit. Ces cartes pourraient après élections servir à $d^{\prime}$ autres fins et d'autres enquêtes statistiques.

Elle permet une localisation précise de différentes entités électorales et des phénomènes en cause. La localisation est sans doute l'objet fondamental, le plus ancien historiquement et le plus important encore actuellement dévolu à la carte. La carte a servi depuis la plus haute antiquité aux déplacements sur terre et sur mer des personnes. C'est d'ailleurs grâce à cette performance qu'elle a pu bénéficier des progrès constants au cours des siècles et atteindre son degré de performance actuel. La carte électorale va pouvoir indiquer les itinéraires de déplacement de la population en direction des centres retenus pour les élections. Ce besoin implique deux éléments d'intérêt, d'une part, une précision et une exactitude aussi grande que possible des levés géodésiques. C'est le propre de la carte électorale que d'offrir au citoyen une certitude quasi absolue quant à la localisation de tel ou tel objet par rapport à des repères connus: cordonnées géographiques, éléments physiques, limites administratives, etc. D'autre part la cartogyhie figure préférentiellement les objets ponctuels qui, par le leur nature sont bien visibles et facilement repérables dans le paysage, sommets, montagnes, cloches d'églises, tours, cheminées, etc... Or ces objets ne présentent souvent qu'un intérêt limité pour d'autres usages. La carte électorale aura l'avantage de les figurer d'une manière telle que ces éléments serviront de points de repères dans les chefs-lieux des territoires des secteurs et seront donc bien figurés.

La carte géographique représente la réalité physique du terrain. Il s'agit en particulier de l'oro-hydrogyhie: relief du sol et cours d'eau. Sans doute parce qu'il est question d'éléments des passages considérés comme stables, plus stables en tout cas que les phénomènes humains; ils ont toujours servi de base pour la représentation des éléments planimétriques. Dans une carte électorale, l'orohydrogyhie a toujours bénéficié d'une attention particulière. Les cours d'eau sont des voies de communications par excellence. Nombreuses limites administratives et même celles de circonscriptions électorales sont faites des cours d'eau ou de grandes lignes de relief. Il y a donc un grand intérêt à les représenter.

Elle assure une omniprésence de la toponymie et de la nomenclature. La 
toponymie ou indication des noms des lieux, complète en fait les informations relatives ou repérables. Sur une carte électorale, on transcrit le maximum possible des noms, eu égard bien entendu à l'échelle: localités, villages, chefs-lieux, secteurs, territoires, districts, provinces, cours d'eau, montagnes, etc...Le but de la nomenclature sur une carte électorale consiste à décoder, à traduire les symboles de la carte au même titre que le texte de la légende. La hiérarchie des écritures en fonction soit de la taille, des localités, soit de l'importance des objets figurés souligne avantageusement la symbolique utilisée dans la carte électorale. Sur total, l'abondance de la toponymie et de nomenclature confère à la carte électorale un avantage décisif.

La carte géographique permet une bonne hiérarchisation des unités électorales. La hiérarchie administrative ou celle des unités électorales se traduit: d'une part grâce à la toponymie citée ci-haut qui transcrit les variations d'importance des chefs-lieux de provinces, des districts, des territoires, des secteurs ou de la commune; d'autre part grâce au report des limites électorales correspondantes. L'existence des limites commerciales jusqu'au 1/100.000, échelle déjà relativement petite se révèle fort précieuse aussi bien pour les études géographiques que pour l'utilisation à des fins électorales.

Elle fait mieux ressortir l'importance des voies de communications et de transport en général. L'importance des élections traduit sans conteste le besoin de se déplacer sur le terrain plus que celui d'étudier la structure et l'importance du réseau. La preuve en est jusqu'à ces dernières années l'accent était davantage porté sur les caractéristiques techniques des voies routières et ferrées, largeur, viabilité, modalités d'insertion dans le paysage, etc... que sur l'importance de leur trafic. D'où cette hiérarchie imposante, en particulier pour les routes. Cette carte indiquera le degré d'accessibilité où l'état des routes dans les sites.

La carte géographique indique l'emplacement des phonies, antennes - relais. Des phonies devront être prévues pour couvrir l'ensemble du territoire national et de le rendre accessible par voie des ondes jusqu'aux petites entités possibles. La carte indiquera l'emplacement de ces phonies et autres équipements nécessaires.

En résumé, les traits généraux de la carte géographique électorale et son rôle absolument indispensable se dessinent déjà à partir des observations faites cidessus. Les échéances électorales dans un pays ont extrêmement besoin de ces éléments pour donner à la population la vraie information et pour même la diriger. Bien sûr qu'en Afrique en particulier, les difficultés auxquelles il faut faire face dans une telle entreprise sont nombreuses et plus importantes en matière d'élection sont celles dues au transport.

La plus grande partie de l'Afrique est couverte des forêts denses et des savanes où l'on ne peut se rendre en véhicule motorisé que pendant la saison sèche, ces régions étant inondées pendant la saison de pluies. Il serait donc préférable que les élections aient lieu en saison sèche. 


\section{Elaboration de la Carte Geographique Electorale}

En matière électorale, il est souhaitable que les autorités chargées de conduire les élections possèdent les cartes géographiques détaillées du pays mais ceci est impossible pour les grands pays, de sorte qu'on est obligé de recourir à des moyens de rechange. Toutefois les cartes dressées pour les élections peuvent se révéler utiles à beaucoup d'égards.

La délimitation du territoire en vue des élections est une opération fondamentale. Trois aspects sont importants et dont il faut tenir compte:

- La détermination exacte des frontières internationales du territoire où les élections doivent avoir lieu.

- La détermination des limites intérieures qui séparent les diverses divisions territoriales pour lesquelles on peut avoir des renseignements sur le chiffre de la population par exemple et celui des personnes en âge de voter. Il s'agit généralement des divisions administratives (provinces, districts, territoires, secteurs, communes, pour le cas de la RDC) censées être légalement délimitées.

- La subdivision des entités territoriales en des unités électorales c.à.d. les plus petites divisions territoriales établies pour les élections.

Les travaux géographiques et cartographiques se feront suivant certaines étapes absolument distinctes et dont il faudra respecter le déroulement. Il s'agit de procéder à l'Inventaire et au rassemblement des cartes et autres renseignements utiles. Il s'agit d'identifier et de rassembler toutes les cartes qui peuvent aider à l'élaboration de la carte électorale. Pour cela, le mieux serait de solliciter le concours de tous les services de l'Etat, de toutes les institutions publiques et privées et d'autres établissements qui peuvent disposer des cartes utiles. L'inventaire est d'intérêt car il dresse le profil cartographique d'un pays. Nous retenons ainsi, par pays une fiche descriptive comprenant deux sections, une générale et l'autre spécifique à la cartographie. Le portrait d'ordre général présente la superficie du pays, la population, la date d'indépendance. Le volet cartographique comprend l'organisme producteur, l'échelle cartographique, le type de carte (théine), l'année de production (ou la période et le nombre de feuilles réalisées en fonction du projet). Dans le pays où aucun service géographique et cartographique n'existe, le premier travail pourrait être effectué par le service cartographique de l'armée, les services de cadastre et les services géographiques locales. L'inventaire fournira des renseignements détaillés sur les cartes existantes en rapport avec les divisions territoriales du pays, les cartes à grande échelle et les plans de grandes agglomérations, zones urbaines et régions à population dense. Les cartes jugées utiles pour les élections, sans modifications ou avec quelques modifications doivent être ressemblées et indexées eu égard à leur valeur relative. Il faudra également recueillir les croquis et photographies aériennes, les images satellitaires pour 
lesquelles il n'existe pas de carte: ainsi que les renseignements sur les distances entre zones habitées. En raison du volume de travail qu'impose le rassemblement et le classement des cartes et autres documents et l'établissement des cartes définitives, il est souhaitable d'entreprendre les travaux géographiques et cartographiques le plus tôt possible.

Etablissement de la carte géographique électorale; si les cartes obtenues par la commission électorale conviennent aux élections sans modifications aucune, on pourra tracer directement sur les cartes les limites des districts et circonscriptions électorales et placer les repères indicatifs. Cependant dans la plupart de cas, les cartes tirées de partout appelleront un travail cartographique. Il faudra alors les modifier soit pour tenir compte des derniers changements des limites territoriales soit ajouter ou retirer des petites zones d'après les résultats des derniers renseignements reçus. Enfin il se peut qu'on soit obligé d'établir des cartes entièrement nouvelles pour les zones qui n'ont pas fait l'objet des levées cartographiques particulières ou celles pour lesquelles les cartes existantes ne conviennent pas aux élections. Cette tâche extrêmement importante sera réalisée par les services cartographiques et géographiques du pays habilité à produire ce genre de documents.

\section{Circonscription Electorale}

La circonscription électorale est la plus petite division territoriale établie pour les élections. Habituellement il est assez réduit pour les habitants appelés à voter y accèdent facilement sans parcourir de longues distances. Le succès et la réussite d'une opération électorale dépendent en gros de la taille de circonscriptions tant en ville qu'en milieu rural. Les limites de chaque circonscription doivent tenir compte du nombre approximatif des personnes en âge $\mathrm{d}$ voter et d'autres facteurs dont a été question ci-haut. Tous les circonscriptions électorales doivent figurer sur la carte. En milieu urbain, le critère principal est vraisemblement le nombre des électeurs que la circonscription pourra recevoir dans le laps de temps prévu. Ce nombre est généralement élevé comparativement au milieu rural compte tenu des conditions de déplacement assez rapide.En milieu rural par contre, il faudra souvent réduire le nombre d'électeurs compris dans une circonscription électorale en raison du temps que l'électeur devra sans doute consacrer pour atteindre son bureau de vote. L'exactitude avec laquelle les circonscriptions électorales sont délimitées sur une carte peut avoir un effet important sur la valeur des élections. Lorsque les limites des circonscriptions sont tracées avec soin et que la population connaît parfaitement les limites de sa circonscription, les risques d'omission $\mathrm{n}$ de fraude, ou de double compte sont semblablement réduits. Chaque circonscription électorale doit être entièrement compris à l'intérieur d'une seule région administrative et non à cheval sur une ligne de démarcation administrative ou politique. Le succès de la limitation des circonscriptions électorales dépend dans une large mesure de la qualité de renseignements géographiques dont on dispose. Dans les centres 
urbains en particulier et dans le localités dotées du même statut que les villes, des plans détaillés avec indication des rues et des bâtiments principaux des circonscriptions. Pour les localités non urbaines, on emploi souvent des répertoires des localités et des cartes à l'échelle indiquant l'emplacement de localités, les routes principales et secondaires reliant les localités voisines ainsi que les cours d'eau, les côtes et d'autres éléments topographiques.

\section{Quelques Details TechniQues}

Dans un exposé comme celui - ci n'est pas possible d'abord les détails techniques de la cartographique mais peut être utile de passer brièvement en revue quelques suggestions auxquelles les cartes utilisées à des fins électorales devront répondre:

- Toutes les cartes doivent être tracées sur du papier calque sensible, papier calque ou astafoil permettant de faire des reproductions photographiques, des tirages ozalides, elles doivent porter l'indicateur de la source des renseignements cartographiques et de la date de leur compilation.

- Chaque carte doit porter une flèche dirigée vers le Nord ainsi que des points de repères indiquant la position du quadrillage géographique et l'échelle horizontale des distances.

- L'échelle de la carte ne doit pas être inférieure à 1/100.000 chaque fois qu'il s'agit de petites divisions administratives. Les renseignements minimum qu'il y a lieu d'indiquer sur les cartes de petites divisions administratives ou des régions rurales utilisées pour les élections sont:

$\square$ les principales caractéristiques physiques accompagnées de leurs noms (cours d'eau, montagnes, plaines, chaînes, plateaux);

$\square$ les limites de communication avec les noms de principaux itinéraires (chemin de fer $\mathrm{n}$ routes et pistes);

$\checkmark$ les limites des divisions et subdivision administrative;

$\checkmark$ l'emplacement et les noms de toutes les agglomérations et localités d'une certaine importance.

L'échelle de cartes des zones urbaines ne doit pas être Inférieures à 1/100.000. Ces cartes doivent fournir au moins les renseignements suivants:

- L'emplacement et les orientations approximatifs;

- les principales caractéristiques physiques avec leurs noms, rues avec leurs noms;

$\square$ les limites de la zone et ses subdivisions;

$\checkmark$ les moyens de communication (chemins de fer, tramways, routes, ponts);

- les terrains publics et leur emplacement (parcs, marchés, cimetières);

$\checkmark$ les zones utilisées à des fins industrielles. 
Si les originaires des cartes ne peuvent être de dimensions égales, il convient d'éviter de trop grandes différences. La dimension choisie doit permettre d'éviter d'avoir à publier ou à rouler la carte pour la conserver, elle ne doit pas dépasser les dimensions des étuis à cartes du modèle courant du commerce. Toute carte qui risquerait d'excéder ces limites doit être divisée en sections.

\section{La Carte GeographiQue et Les Elections a Travers Le Monde}

Les élections ont besoin dans leur phase préparatoire de Cartes même anciennes surtout lorsqu'elles indiquent les principales caractéristiques physiques et écologiques. L'utilisation des cartes anciennes comme documents de travail constitue une économie d'argent et de temps. On y trouve des renseignements sur les conditions résultats du milieu naturel, la répartition de la population, les communications et les ressources.

Les rapports administratifs sur les élections effectuées dans un certain nombre de pays développé montrent que des pays de plus en plus nombreux utilisent davantage de renseignements cartographiques pour la préparation de leurs élections. Un des meilleurs exemples de la façon dont les électeurs peuvent stimuler l'intérêt porté à la cartographie et aux travaux dans ce domaine est fourni par les dernières élections présidentielles organisées en France. Les régions, les départements, les arrondissements, les communes, tout avait été cartographié.

En Iran, à l'occasion des dernières élections, les cartographes avaient été utilisés et envoyés partout sur les lieux pour déterminer l'emplacement de tous les villages du pays et pour dresser les cartes des villes et des villages de plus de 5000 habitants.

De même à l'occasion des récentes élections au japon, on s'est spécialement attaché à préparer des cartes des districts électoraux qui ont ensuite servi d'unités pour diverses enquêtes par sondage. Pour les élections de 1999 en Afrique du Sud, chaque bureau de vote était porteur d'un plan ou d'un croquis indiquant les limites électorales ou d'une description écrite de ces limites.

Les élections à différents niveaux dans un pays sont généralement organisées après 4,7 ans suivant le système en place. Dans beaucoup de pays, leur préparation nécessite l'utilisation et la révision des cartes dont on dispose déjà et l'établissement de cartes poser pour les régions qui ne sont pas encore comprise dans la documentation cartographique existante. C'est dire qu'en matière des élections une collaboration très étroite entre cartographes et organisateurs des élections est nécessaire. Une meilleure coordination entre d'une part les divers organismes chargés de l'établissement et de la conservation des cartes administratives, routières, sanitaires, agricoles et d'autre part le service chargé de préparer et d'exécuter les élections permettrait une utilisation plus économique et plus rationnelle. Si les cartographes se familiarisent avec les normes techniques des renseignements cartographiques destinés aux électeurs, les cartes pour les élections ne pourront que devenir meilleures et plus nombreuses. 


\section{Les Travaux Cartographiques Après les Elections}

Alors que la plus grande partie des travaux cartographiques a lieu au courant de la phase préparatoire, il y a des tâches supplémentaires à exécuter après les élections, période au cours de laquelle on a l'habitude de recueillir un grand nombre des renseignements géographiques et cartographiques nouveaux. Il sera souvent demandé aux agents affectés dans les bureaux de vote d'indiquer à l'issue des élections, toute modification ou addition qu'ils jugent nécessaire d'apporter aux cartes de leur district ou circonscription électorale. Au cas où il n'a pas existé lors des élections de carte appropriée, on pourra demander aux agents de réaliser des croquis, des schémas des circonscriptions des circonscriptions où ils ont été affectés. Tous ces renseignements doivent être reportés sur les cartes officielles au bureau central. L'établissement après élections, des cartes corrigées, des cartes sommaires, des graphiques est dans un pays, le domaine réservé à l'Institut Cartographique Officiel. C'est d'ailleurs la même institution qui est censée élaborer en amont la carte électorale. En RDC, cette prérogative est dévolue à l'Institut Géographique du Congo qui a pour mission fondamentale l'élaboration de la carte de base à 1/50.000. A cette mission s'ajoute:

- L'exécution des travaux géographiques, topographiques, photographiques, cartographiques et de couverture aérienne sur l'ensemble du territoire national.

- L'organisation de la recherche destinée à l'amélioration des méthodes de production des cartes géographiques.

- La constitution d'une banque de données géographiques de l'ensemble de la RDC.

- La conservation des documents cartographiques ainsi que des informations géographiques du pays.

Dans certains cas, on fera appel à ce même service pour dresser d'autres graphiques et diagrammes à figurer dans les publications, ainsi que pour les travaux de dessin à l'occasion des travaux des élections.

Enfin, il est utile que des dispositions soient prises pour conserver les originaux des cartes pendant les intervalles entre les élections. Avant de les classer en vue d'une rentabilisation future, on réparera ou on recopiera les cartes qui auraient été endommagées.

D'ordinaire dans les pays organisés, les services officiels en matière cartographique continuent à corriger les anciennes cartes et a en établir de nouvelles pendant l'intervalle entre deux échéances électorales.

\section{Autres Methodes Geographiques}

L'observation de la situation cartographique à travers le monde montre qu'il existe encore des régions dans lesquelles on ne trouve que des cartes peu détaillées voir 
pas de cartes du tout. Dans de nombreux pays africains qui font partie de ce groupe, il est même très difficile de dresser des cartes à cause du manque d'équipement ou en raison de divers obstacles pour atteindre les coins reculés. En outre un certain nombre de pays n'ont pas de cartes simplement par manque de fonds, du personnel ou du matériel nécessaire pour créer un service cartographique national pouvant également s'occuper de la carte électorale.

En pareil cas, il faut s'en remettre à un répertoire systématique général de toutes les localités ou villages, de tous les secteurs administratifs, des territoires, des districts, des provinces, des communes, des quartiers, etc.

L'établissement de répertoire de ce genre est une tâche de longue haleine qui ne se limite pas seulement aux pays où les cartes sont rares. Bien au contraire les répertoires sont utilisés très souvent, en plus de la documentation cartographique, dans les régions agricoles où la population est très clairsemée. De toute manière, il faut dans tous les cas s'assurer s'il y a véritablement cohérence entre les renseignements figurant sur les cartes et sur les répertoires et, le cas échéant, corriger les répertoires et les cartes. Dans de pays qui ont l'habitude d'organiser leurs élections sur base de carte électorale et qui possèdent beaucoup de renseignement cartographiques, l'établissement des répertoires de localités et des circonscriptions électorales se borne à une mise à jour des données existantes.

L'utilisation de la carte à des fins électorales peut être complétée par celle de la photographie aérienne ou des images satellitaires. La photocopie aérienne a ses avantages dans la mesure où elle fournit de détails que les cartes ordinaires mais tel n'est pas notre propos dans le présent exposé. L'utilisation de ces techniques accroît et donne aux élections plus de chance de réussite.

\section{CONCLUSION}

La carte géographique électorale est un document d'une grande valeur dans l'organisation du recensement et des électeurs dans un pays. Elle demeure plus que jamais un remarquable outil de travail dont on ne saurait se passer.

Le développement de nouvelles techniques et méthodes électorales, loin de lui porter ombrage lui fournira au contraire un nouveau champ d'investigation et la chance d'accomplir de nouvelles performances tant sur le plan de la perception technique que sur celui de la visite de récolte d'information.

La carte géographique électorale permet de visualiser les phénomènes de présenter les faits que la population appelait à voter est censée connaître et maîtriser. Elle est un document guide, un cadre de référence qui circonscrit l'espace, le territoire à l'intérieur duquel les opérations électorales ont eu lieu. La carte électorale donne des précisions sur la superficie et l'étendue du territoire, les subdivisions ou circonscriptions au sein desquelles vont pouvoir se dérouler les mouvements des populations. Chaque électeur est censé évolué dans le périmètre lui défini et dont il peut prendre connaissance. Ce qui lui exige une certaine discipline dans les mouvements et dans l'accomplissement de son devoir civique. 
Hormis ces caractéristiques fondamentales, la carte géographique des élections fixe également le pouvoir sur le volume de la population par circonscription électorale, localise le cas échéant les bureaux de vote, les itinéraires surtout en milieu rural, les distances à parcourir. La population doit savoir avec la plus grande exactitude où se situe tel bureau de vote, tel passage à niveau, tel point ou telle intersection de routes importantes, surtout que certes dont pouvoir lui servir dans ses déplacements sur terrain.

Loin de se tenir à l'écart des progrès techniques, la carte géographique électorale est aujourd'hui la base de toute opération en la matière. Les institutions géographiques ou l'Etat ont dans chaque pays le monopole de mon élaboration. Il est dès lors recommandé au pouvoir public de doter ces institutions des moyens et un équipement adéquat leur permettant de bien jouer leur rôle électoral. 